\title{
Contents, Vol. 29, Supplement 1, 1997
}

\section{Table of Contents}

About JERMOV

Abstracts of Posters $001-160$,

exhibited on Thursday and Friday Abstracts of Posters 201 - 342,

exhibited on Saturday and Sunday Abstracts of Oral Presentations

Sessions on Thursday

Sessions on Friday

Sessions on Saturday

Sessions on Sunday Author Index

8

13

54

91108124146159

7

\section{About JERM OV}

The Joint European Research Meeting in Ophthalmology and Vision is a joint effort by the AER (Association for Eye Research), the ECORA (European Community Ophthalmic Research Association), the European Chapter of ISCEV (International Society for Experimental and Clinical Electrophysiology of Vision) and the OOG ( Ophthalmic Oncology Group) in collaboration with other Special Interest Groups such as the FAN (Fluoangiography) Club, the European Cornea Conference, the ESA (European Strabismological Association) and others. The meeting is open to all and intends to cover all fields of research in Ophthalmology and Vision. Since 1994 it is organized every year in October.

Organizing Committee of JERMOV 97

President G. SOUBRANE, France

Secretary-Treasurer L. MISSOTTEN, Belgium

Members N.R. GALLOWAY, United Kingdom

J.U. PRAUSE, Denmark

A. WEGENER, Germany

The complete schedule of the sessions is available on the Web site:

http://www.jermov.org/jermov/

8

rremiere aans le $<$ aiaucome :

la voie uvéosclérale 


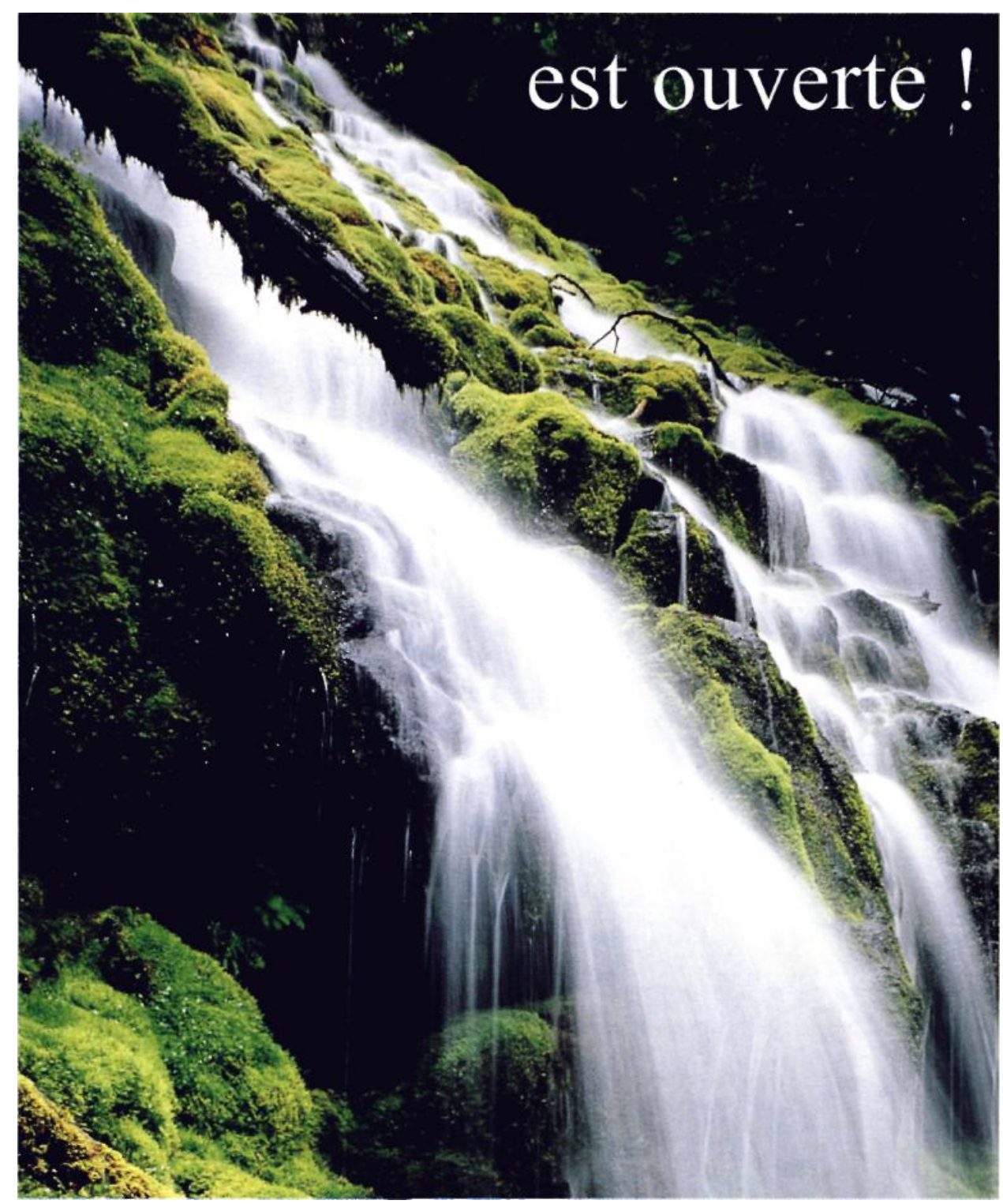

Pharmacia \& Upjohn signe la Revolution des Prostaglandines $1 / 8 \mathrm{j}$

Pharmac¡a\&Upjohn B.P. 210, 78051 St-Quentin-Yvel¡nes Cedex. Tél.: 01.30.64.34.00 · Fax : 01.30.48.01.49

DENOMINATION DU MEDICAMENT: TIMOPTOL IP 0,25 POUR CENI, collyre. TIMOPTOL' LP 0,50 POUR CENT, collyre.

COMPOSITION QUAUTATIVE ET QUANTITATIVE: Maléate de timolol (DCI), 0,25\% : $3,42 \mathrm{mg}, 0,50 \%: 6,84 \mathrm{mg}$. Quantité correspondent á timolol base, 0,25\%: 2,50 mg, 0,50\%: $5,00 \mathrm{mg}$. gomme gellone, $0,25 \%: 6,00 \mathrm{mg}, 0,50 \%: 6,00 \mathrm{mg}$. mannitol, 0,25 \%: 45,00 $\mathrm{mg}, 0,50$ $\mathrm{X}$ : 40,5 mg. trométamol, 0,25\%: 0,91 mg, 0,50\%: 1,82 mg. bromure de benzododécin ¡um, 0,25 $\%: 0,12 \mathrm{mg}, 0,50 \%: 0,12 \mathrm{mg}$. eau pour preparations injectobles qsq, 0,25\%: $1 \mathrm{ml}$., 0,50 X: 1 ml..

FORME PHARMACEUTIQUE: Collyre 
DONNÉES CLINIQUES : 4.1 Indications thérnpeutiaaes: - Hyperton ${ }^{\mathrm{e}}$ intro-oculnire. Glaucome chronique à angle ouvert. - Glaucome de I'aphaque. 4.2 Posologie et mode d'aom; $\pi$ istrnlion: · Agiter le flacon, une fois énergi-auement, avant Tinstillation. Monolhérapie: » Debater le traitement gar I'instillation d'l goutte de collyre à 0,25\% dans I'æúl malade, 1 fois par jour. ' En cas d'efficacité insuffisante, passer au dosage $0,50 \%$ à raison d'l goutte par jour dans $1 \frac{1}{8} 1$ malade. Association: Pour oblenir un résultat maximal, 1Ophtalmologiste pourra associer à ce collyre, s'il le 'uge nécessoire: · soil un collyre ontiqlaucomateux sympat $3 / 80$ ou para-sympathom;mét;que, a soit un traitement par voie generate ó I'aide d'un inhíbiteur del'anhydrase carbonique. Dans ces cas, les autres collyres doivent êlre odmi-

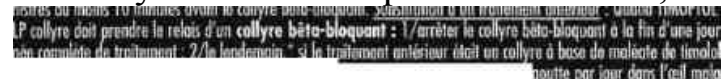

le remplacer par TIMOPTOL" LP ò la même concentration, (si disponlle) ó raison d'l de,' si le traitement antér ¡eur était un collyre à base d'un autre bêta-bloquant, le remp collyre ó raison d'l goutte par jour dans I'æil malade; 3/en cas d'efficacité insuffisante, passer au TIMOPTOL" LP $0,50 \%$, collyre à raison d'l goutte par jour dons I'æ 1 malade. · Quond TIMOPTOL" LP doit prendre le relais d'un collyre ant;-glaueomnteux autre que bélo-blOquanl: I/superposer les deux trailements pendant une ¡ournée en adoptant la posologie d'l goutte par jour de TIMOPTOL LP $0,25 \%$, collyre dans I'æ;1 malade; $2 /$ le lendemain, suppr $;$ mer le collyre anti-glaucomateux antérieor, et poursuivre le traitement ovec TIMOPTOL LP $0,25 \%$ collyre, seul, $\alpha$ raison d'l goutte par jour dans 11/8il molade; 3/en cas d'efficacité insuffisante, posser ou TIMOPTOL* LP $0,50 \%$, collyre à rai-m d'l goutte par jour dons I'æ 1 malade. · Quand TIMOPTOL LP collyre doit se subst;tuer $\alpha$ plusjeurs antiglaucoma-teux associés, le médecin peut, en fonction du cas, en ¡nferrompre certains au decider de les arrêter tous. Mais les suppressions ne doivent impliquer qu'un seul medicament $\alpha$ la fois. Lorsque les malades passenl des myot;ques $\alpha$ TIMOPTOL* LP collyre, un exnmen de la refraction peut s'avérer nécessa;re, quand les effets des myot;ques ont $d_{i}$ spa-ru. Vejller à ce que le malade se conforme strictement á la prescription medicate.

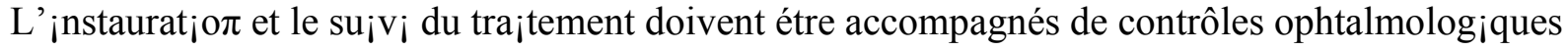
réguliers (et notamment de celui de la pression jntro-oculai-re). 4.3 Contre-i $\pi$ dicotions: II convient de garder ò [esprit les contre-indicatíons des bêta-bloquants administrés par voie générale, $b_{j}$ en qu'elles ne soient observées que de façon exceptionnelle oprès administration par voie ocula;re: -osthme et bronchapneumopathies chron;ques obstructives, - ¡nsuffisance cardiaque non contrôlée par le traitement, -choc cardiagén;que, - blocs aurjculo-ventriculaires de second et troisième degré non appareillés, - nngor de Prinzmetal, -malodie du sinus (y campris bloc sino-auricula;re), -bradycardie ( $<45-50$ tontractions/min.|, -phénomène de Raynaud et troubles circulatojres périphériques, - pheochromocytome nan trajté, - hypotension, - hypersensibjlité au timolol, ou ó tout produit ou traitement aya $\pi \dagger$ auparavanl provoqué une reaction onaphyloct ique, - association à la floc-tafénine (cf. Interactions médicamenteuses). Ce medicament est généralement déconseillé en ossociation ó I'am;odaro-ne. 4.4 Mises en garde et precautions d'emploi : İ/Oailoires: - line surveillance particulière doit être exercée chez des patients pre $\pi$ ont un bêta-bloquant par vole arale en plus de TIMOPTOL* LP collyre: afin d'éviter une potentialisation: - au niveau du tonus oculajre. - au njveau des effets systémiques. Dans le cas ov́ TIMOPTOL LP", collyre est admin ${ }_{j}$ s-tré pour $\mathrm{d}_{i} \mathrm{~m}_{i}$ nuer la pression $¡$ ntra-oculaire chez les patients ayant un glaucome par fermeture de I'angle, un myotique doit être assacjé. En effet, chez ces pat;ents, I'objectif ¡mmédiot du traitement est la réouverture de I'angle, ce qui necess;te I'emploi d'un myot;que afjn d'obten;r une constriction pupillajre, le maléale de timolol n'oyant pas ou peu d'effet sur la pup;lle. - Porteurs de lent;lles de contact: ce collyre n'a pas été étudié chez ces patients. - Utilisation chez I'en-fant: ce collyre n'a pas été étudié chez le 
premature, le nouveou-né et I'enfont. 2/Généroles: li convient de garder à l'esprit les mises en garde et precautions d'emploi des bêta-bloquants administrés par voie générale, $b_{j}$ en qu'elles ne soient observées que de façon except ${ }_{j}$ onnelle oprès instillation oculajre: $\mathrm{Ne}$ ¡amajs jnterrompre brutalemenl le trajtement chez les ang;neux: I'arrêt brusque peut entraîner des troubles du rylhme graves, un ¡nfarctus du myocarde ou une mart sub;te. ['attention des sportifs est attirée

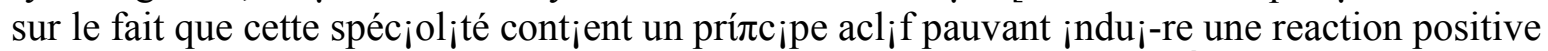
des tests pratiques lors des contrôles anlidopage. Precautions d'emploi: Árrêl du Irajtemenl: Le traitement ne doit pas être ¡nterrompu brutalement, en particul ${ }_{j}$ er chez les pat ${ }_{j}$ ents présentant une

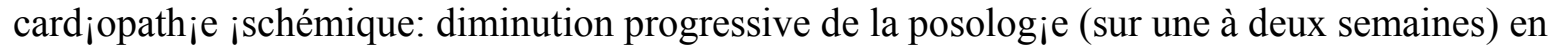
commençant en même temps, si nécessoire, le trajtement subst $j_{i}$ tutif, pour éviter une aggravat ${ }_{j} a n$

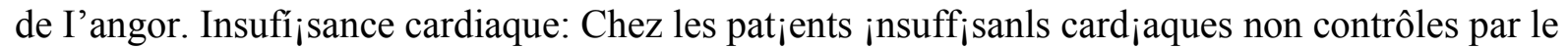
traitement: ne pas ut $i_{i} i_{\text {ser de }}$ beta-bloquants. Bradycardie: $S_{i}$ la frequence s'abo $j$ sse au dessous de 50-55 pulsations par minute au repos et que le patient présente des symptômes lies à la bradycardie : diminuer la posologie. $1 / 8$ c aurjculo-ventriculaire $1 / 8$ premier degré: Etant donné I'effet dromotrope négatif des bêta-bloquants, ceux-c; ne doivent être administrés qu'avec prudence nux pat;ents présentant un bloc aurjculo-ven-triculajre du premier degré. Pheochromocytome: I'utilisat;on des bêta-bloquants dans le trajtement de [hypertension due ou pheochromocytome trajté necess ;te une surveillance étroite de la press;on artérielle. Sviet; âgés, insuH $\mathrm{H}_{i}$ sants rénoux et/mi hépatiques: Chez ces sujets $\alpha$ risque et quand le medicament est administré par voje générale: adaptation pasologique souvent nécessajre. Sviets diabétiques: Préven ${ }_{i}$ r les malades el renforcer en debut de trajtement I'au-tosurvejllance glycém;que. Les signes annoncialeurs d'une hypoglycémie peuvent être masques, en particulier tachy-cardje, palpitations et sueurs. Reactions ahrgipes: Psoriasis: possible augmentation de la sens $; b_{i} l_{i}$ té aux allergènes et aggravation des manifestations anaphylactiques par les bêta-bloquants. Chez les patjents suscept;bles de fa;re une reaction anaphyloct;que severe, quelle qu'en soit I'orig;ne, en part;culier ovec des prowls de contraste ¡odés ou la floe tafénine (cf. Interactions médicamenteuses) au au cours de trajtements désensioj $T_{i}$ sants: aggravation possible de la reaction et resistance à son trajtement par [adrenaline aux posologies habituelles. Anesthesia générale: les bêta-bloquants vont entraîner une attenuation de la tachycardie réflexe et une augmentation du risque d'hypotens;on. La pour-suite du trajtement par bêta-bloquant $d_{j} m_{j}$ nue le risque d'orythm;e. d'ischém;e myocardique et de poussées hypertensives. II convient de préven $j$ I I'aneslhés $j$ ste que le patient est trajté par un bêta-bloquant. $S_{i}$ I'arrêt du traitement est

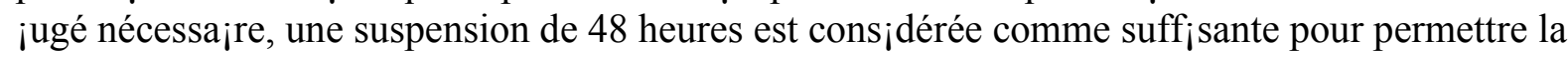
réappar $i_{i}$ on de la sens $; b_{i} l_{i}$ té aux catécholamínes. Dans certains cas le trajtement bêta-bloquant ne peut être ¡nterrompu: - chez les malades atte ¡nts d' ${ }^{\prime}$ nsuffisance corona ¡re, ¡ 1 est souha¡table de poursuivre le trajtement ¡usqu'á [intervention, étant donné le

,.$j$ appel à des produ $i$ ts auss $i$ peu dépresseurs myocardiques que possible et les pertes sanguines devront être compensées. L'attention des sport;fs est attirée sur le fait que cette spécjalité contient un

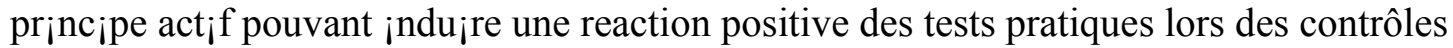
antidopage. 4.5 Interactions avec a'autres medicaments et autres formes d'interoctions: 1/Oculaires: Une surveillance ophtalmolog;aue est nécessajre en cas de trajtement concomitant par un collyre contenant de [adrenaline (risque de mydriase). 2/ßénérales: $B_{j}$ en que les quanlités de bêta-bloquants passant dans la circulation systém;que sajent faíbles oprès instillation oculajre, le risque d'¡nteractjons médicamenteuses ex ¡ste. II canvient done de tenir compte des 
interactions observées avec les bêta-bloquants administrés par voje générale: Association contre¡ndiquée: + Floctafénine: En cos de choc ou d'hypotens jon dus á la floctafénine.

vo¡e générale: Association contre-indiquée: + Floctafénine: En cas de choc ou d'hypotensjon dus $\alpha ́$ la floctafénine, reduction des reactions cardíovasculojres de compensation par les bêtabloquants. Association déconseillée: + Amiodorone Troubles de la contract; $1_{i}$ té, de I'automatisme et de la conduct;on (suppression des mécan ¡smes sympa-th¡ques compensateurs). Associations nécessitant des precautions d'emploi: + Anesthésiques volot;ls halogénés Reduction des reactions cardio-vasculajres de compensation par les bêta-bloquants. (L' ¡nhibition bêta-adrénerg ique peut être levée durant [intervention par les bêta-stimulants). En regie générale, ne pas arrêter le trajtement bêta-bloquant et de loute façon, éviter I'arrêt brutal. Informer

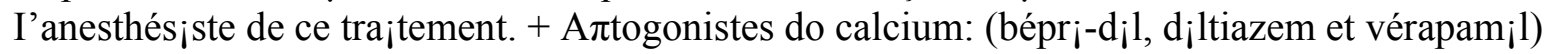
Troubles de I'automot;sme (bradycardie excessive, arrêt sinusal), troubles de la conduct;on aur $;$ culovenlr; $;$ culaire et défaillance cardiaque (synergie des effets). Une telle association ne doit se fajre que sous sur-

veillance clinique et ECG étrojte, en particuljer chez le sujet âgé ou en debut de trajtement. +

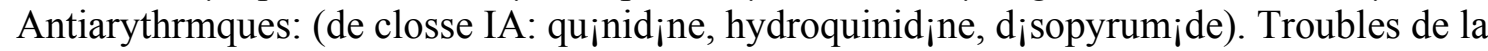

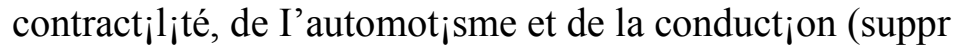

Boclofène: Majorat;on de $\Gamma$ effet a $\pi \mathrm{t}$; hypertenseur. Surveillance de la press;on artérielle et adaptation posologique de 1'ont;hypertenseur si nécessoire. + Clon;i ine: Bêla-bloqvants non cardiosélect;ls: déajt pour le propratolol et le nadolol. En cas d'arrêt brutal du traitement par la clonidine, augmentation importante de lo pression artérielle avec $\mathrm{r}_{j}$ sque d'hémorragie cérébrale par effet sympathom;mét;que. Arrêter de façon progressive la clonidine. + insuline, sulfamides nypoglycém;iants: Tous les bêta-bloquants peuvent masquer certains symptômes de [hypoglycémie : les palpitations et lo tachycard;e. Préven;r le malade et renforcer, surtout ou debut du trajtement, I'autosur-vejllance sanguine. + Lidocaï̈e: Décrit pour le propranolol, le métoprolol, le nadolol. Augmentation des laux plos-mut ques del $_{i d o c a i ̈ n e}$ avec majorat;on possible des effets indésjrables neurologiques et card;aques (diminution du métaool;sme hépat;que de la lidocaïne).Adapter la posolog ${ }_{i}$ de la $1_{j}$ docaïne. Surveillance clinique, étectrocardiogra-phique et, éventuellement, des concentrat;ons plasmatiques de $1_{j}$ docoïne pendant le trajtement bêtabloquant et après son arrêt. + Produits de contraste iodés: En cas de choc ou d'hypotension dus aux produjts de contraste jodés, reduction par les bêta-bloquants des reactions cardio-vasculajres de compensation. Le traitement par le bêta-bloquant dolt être arrêté chaque fois que cela est possible avant [exploration radiologique. En cas de poursu;te indispensable du J tés. + Propafénone: Troubles de la contract;AINS pyrazolés). + A $\pi$ tagonistes do calcium: dihydropyi idines (amladipine, félodipine, lacid $\left._{i} p_{i} n, n_{i} f_{\text {fédip }} n_{n}, n_{i} c d_{i} p_{i} n_{e}, n_{i} \bmod _{i} p_{i n e}, n_{i t r e n d} p_{i} \pi e\right)$. Hypotension, défaillance cardiaque chez les malades en ¡nsuff; sance card;oque laten-te ou non contrôlée (effet ¡nolrope négat; $f_{i n}$ vitro des $d_{i}$ hydropyr ${ }_{i} d_{i}$ nes, plus ou mojns marque en fonct;on des pro-d bêta-bloquant peut par ajlleurs $m_{j} n_{i} m_{j}$ ser la reaction sympath $;$ que réflexe $m_{j}$ se en $j$ eu en cas de repercussion hémody-namique excessive. + Antidépresseurs imlpraminiques (triøyclíques), neorolepfiqoes: Effet ontihypertenseur et risque d'hypotens;on orthostat;que mojorés (effet $\left.\operatorname{add}_{i} t_{i} f\right) .+$ Cort $i c o i ́ d e s$, tétracosactide (voie générale): Diminution de [effet ontihypertenseur (retention hydrosodée des corticaïdes). + Méiloqoine: $\mathrm{R}_{i}$ sque de bradycardie (addition des effets bradycardisants). 4.6 Grossesse et allaitement: Grossesse: Aspect léralogène: Cfiez [animal, aucu-ne action teratogène n'a été $m_{i}$ se en evidence. Dans I'espèce humajne, aucun effet tératoqène n'a été ropporté ó ce ¡our et les résultats d'études prospectives contrôlées n'ont pas 
fall état de molfarmat;ons óla najssance. Aspect foeto-tox ¡que: Les bêta-bloquants diminuenl lo perfusion placentaire, ce qui peut entraîner la mart du foetus in utero ains i que des accouchements avant terme et prematures. Aspect néo-nalal: Chez le nouveau-né de mere trajtée, [action bêta-bloquante pers $j$ ste plusjeurs jours après la najssance: $s i$ cette rémanence peut être sans consequence clinique, il est néanmoins possible que survienne une défaillance cardiaque nécessitant une hosp;tal;sat;on en soins intens;fs (cf. Surdosage), en évitant les solutes de remplissage (risque d'OAP); par ajlleurs bradycardie, détresse resp ¡ratojre, hypoglycémie ont été signajèes. C'est pourquoi une surveillance attentive du nouveou-né (frequence cardiaque et glycémie pendant les $3 \alpha$ á 5 premiers jours de vie) est recommandée en milieu specialise. Allaitement: Les bêta-bloquants son) excretes dans le lajt (cf. Propriétés pharmacocinétiques). Le $r_{i}$ sque de survenue d'hypoglycém;e et de bradycardie n'a pas été évalué; en consequence et par precaution, I'allajtement est déconseillé pendant la durée du traitement. 4.7 Effets sur Taptitude $\alpha$ conduire des véhicules et ó utiliser des mnchines: Une vision trouble transitojre, durant géné-ralement 30 secondes à 5 minutes, peut se produ;re ò la sujle de I'¡instjllotion, et des troubles éventuels de la vision peuvent $d_{i} m_{i}$ nuer [aptitude ä réal ${ }_{i}$ ser des laches dangereuses telles que I'utilisat;on de machines ou la conduite automobile. 4.8 Effets indésirobles: Ì/Ocultíres: Poss ¡bilité de: · vision trouble transitojre due à la transformation du produ it en gel après instillation dans $1 \frac{1}{8} 8 \mathrm{il}$; · troubles visuels comprenant des modifications de refraction (dues porfois à I'arrêt du trajtement par les myot;ques), diplopie et ptosis; · douleur, sensation de brûlure et $p_{i}$ cotements, sensat;on de corps étranger, démangea $j_{\text {sans, larmojement; · }}$

con $_{j} a_{n} t_{j} v_{i}$ te, secrétjon, blépharjte, kératite, hypoesthèsje cornéenne. 2/Généraux: II convient de garder $\alpha$ l'esprit les effets indésirobles des bêta-bloquants administrés par voje generate, $b_{j}$ en qu'ils ne soient observes que de façon except;onnelle après instillation oculajre. Au plan clinique: Les plus fré-quemment rapportés: - asthénie. - refroidissement des extrém;tés. bradycardie, severe le cas échéant. - insomnie, cauchemars. - troubles digestifs (gastralgies, nausées, vom ¡ssements). - sécneresse oculajre. Beoucoup plus rorement: - ralentissement de la conduct;on auriculo-ventr $;$ culajre ou intensification d'un bloc aur $;$ culo-ventr ${ }_{j}$ culajre existant. ¡nsuff ¡sance cardiaque. - chute tensionnelle. - branchospasme. - hypoglycémie. - syndrome de Raynaud. - aggravation d'une claudication intermittente existante. $-\mathrm{d}_{j}$ verses manifestations cutonées y (Maoris eruptions

id'cnt;corpsunt;nucléa;resnes'ałcompagnontqu'ex-

cept;onneltemenl de man;festat;ons $\operatorname{cl}_{i} n_{i}{ }_{i q u e s}$ ä type ae syndrome lupique et cédant à I'arrêt du traitement.

d'une claudication ¡ntermittente existonte. - diverses manifestations cutonées y plan bioloqique: On a pu observer, dans de rares cas, [apparition d'onticorps ant... cept;onneltemenl de man;festat;ons $c_{i} n_{i}{ }_{i q u e s}$ ä type ae syndrome lupique et cédant à I'arrêt du trajtement.

4.9 Surdosage: $B_{i}$ en que les quant;tés des bêta-bloquants passant dans la circulation systémique sojent fajbles après

instillation oculajre, le risque de surdosage do¡t être garde ó I'espril et la condujte à ten ¡r caiquée sur cclle habjtuelle-

ment adoptee pour les bêta-bloquants.

PROPRIÉTÉS PHARMACOLOGIOUES : 5.1 Propriétés pharmacodynomiques: BETABIOQUANT NON CARDIO-SELECTIF - Sur le plan general: Le timolol se caraclérise par Irojs propriétés pharmacologiques: - bêta-bloquant non cardiosélectif - absence de pauvo¡r agoniste port;el (ou absence d'actjvité sympathom;mét;que ¡ntrinsèque - (ASI) - effet 


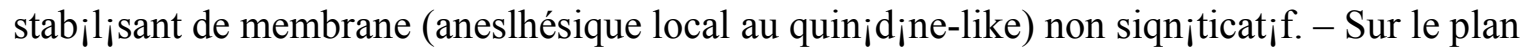
oculajre .'TIMOPTOL*' LP, collyre est une solution opbtalm; ${ }^{*}$ que stérjle de maléate de timolol. Elle abajsse la tension ¡ntra-ocula;re élevée, associée ou non à un glaucome. L'activité de ce collyre est hobituellement ropide, se manifestant environ 20 minutes après ins filiation oculajre. Lo reduction max ¡maje de la pression ¡ntra-oculajre survient en deux ó quatre heures. Ua abajssement $s_{i} g n_{i} f_{j}{ }_{i} t_{j} f$ de la pressjon intra-oculajre est obtenu pendant 24 heures avec les concentrations $0,25 \%$ et $0,50 \%$. í excipient de ce collyre (gomme gellaue) a la propriété de former un gel clajr, transparent $\alpha$ des concentrat;ons basses de polymères en presence de cations. Cette transformation a lieu lors du contact avec le film aqueux précornéen. Cet excipient augmente le temps de contact du medicament avec I'æil ce qui permet de rédujre le rylhme

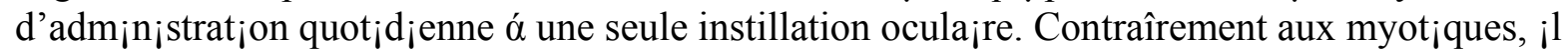
n'y a pratiquement pas de $\bmod _{i} f_{j}$ cation du diamètre pupillajre ou de [accommodation. On n'observe done pas de sposme de [accommodation, ni de modification de I'acujté visuelle. Comme avec les autres drogues antiglaucomateuses, une diminution de la récept ¡vité au maléate de timolol après traitement prolongé a été rapportée chez quelques patients. Cependant, d'après des etudes cliniques menées avec le maléate de t;molol sous forme de collyre chez 164 pat;ents

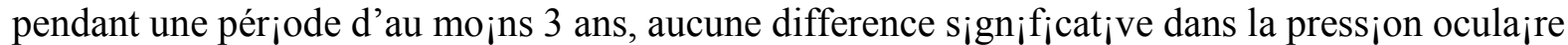
moyenne n'a été natée après la période ¡n $n_{i} t_{i}$ ale de stabilisation tensionnelle. 5.2 Propr $; e ́ t e ́ s$ pharmacocinét;ques: Comme ovec les autres collyres, le timolol associé ó de la gomme gel-lane posse dans lo circulation générale. Une à deux heures oprès instillation d'une goutte de TIMOPTOL PL", un pic de concentration plasmatique est observe. Les pics observes après instillation d'une goutte $\alpha$ ' $0,50 \%$ de timolol sont en moyenne plus fajbles avec le t¡molol assocjé $\alpha ́$ la gomme gellane $(0,28 \mathrm{ng} / \mathrm{ml})$ qu'avec le timolol en solution $(0,46 \mathrm{ng} / \mathrm{ml})$.

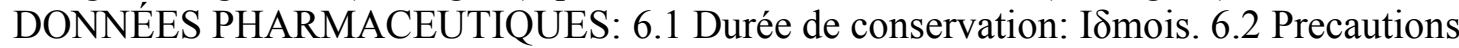
particulières de conservation . A conserver à une temperature inférieure ó $25^{\circ} \mathrm{C}$. A ut $i_{i} i_{i}$ rer dans le mois après ouverture du flacon. 6.3 Nature et contenonce du recipient. Flacon contenant 2,5 $\mathrm{ml}$ (polyethylene basse densité) muni d'un embout compte-gouttes doseur et d'un bouchon en polypropylene bleu clair (TIMOPTOL* LP $025 \%$ ) ou d'un bouchon en polypropylene bleu moyen (TIMOPTOL* LP 0,50 \%). L'embout et le bouchon bleu clair ou bleu moyen sont proteges par un capucnoa transparent et jetable.

PRESENTATIONS ET NUMÉROS D'IDENTIFICATION ADMINISTRATIVE: TIMOPTOL LP 0,25 \%. 337339 - 7:2,5 ml en flacon (polyethylene). Prix: 57,00 F. Remb. See. Sot $65 \%-$ Collect. TIMOPTOL*" LP 0,50 X. 337340 -5:2,5 ml en flacon (polyethylene). Prjx: 60,80 F. Remb. See. Soc. $65 \%$ - Collect.

CONDITIONS DE DELIVRANCE: 1 ; ste I.

TITULAIRE DE L'AUTORISATION DE MISE SUR LE MARCHÊ: Laborato;res MERCK SHARP \& DOHME-CHIBRET - 3, Avenue Hoche - 75114 PARIS Cédex 08. Tél.: 01.47.54.87.00

10. DATES D'APPROBATION/RÉVISION: Date d'approbation: 12.04.94. Date de revision: 5.07.95. -17.03 .97$

Mois

Pour simplifier le traitement

Le 1er collyre beta bloquant à une seule goutte par jour ABSTRACTS 
The abstracts are arranged in order of their numbers in the programme book. Abstracts of posters have three-digit numbers. They are grouped according the subject. Abstracts of oral presentations have four-digit numbers and are arranged per session.

The abstracts are photographic reductions of the submitted abstract forms. The authors are sole responsible for their contents.

12 